\title{
EL SECTOR ENERGÉTICO COLOMBIANO: EL CASO DE LAS ZONAS NO INTERCONECTADAS VISTO A TRAVÉS DE LA INNOVACIÓN LIGERA
}

(Versión preliminar 20-01-17)

Autores:

Carlos Ariel Ramírez Triana Ph.D.

María Alejandra Pineda Escobar M.Sc.

Sergio Alberto Mora

Mauricio Cano.

\section{INTRODUCCIÓN}

Las necesidades energéticas de un país, en conjunto con la forma en la ellas se suplen, determinan en gran medida las posibilidades de desarrollo que tiene el mismo. Sin embargo, no basta con simplificar el problema a un balance entre oferta y demanda energética, sino que un análisis en donde se contemple el tipo de fuentes y usos que se le dé a la misma permite identificar fortalezas en varios frentes, así como también la detección de alertas que pueden contribuir a atender carencias que enfrentan algunos sectores de la sociedad, a través del refinamiento de políticas que surgen de los tomadores de decisiones.

En este documento, se hace una primera aproximación a la provisión de energía para zonas no interconectadas a la malla eléctrica nacional en Colombia con el propósito de, en una segunda entrega identificar el vínculo de este tema estudiado desde la óptica de innovación ligera.

Para lo anterior se dará un marco general de la estructura energética nacional y posteriormente una reflexión más detallada del nivel de cobertura en la red eléctrica, para identificar de esta forma las zonas interconectadas y las formas como, desde lo institucional se aborda, esta problemática.

\section{MARCO GENERAL}

Colombia es un país ubicado en la región noroccidental de Suramérica. Tiene una población cercana a los 49 millones de habitantes. Cuenta con una extensión territorial de 1'141.748 km². Tiene acceso a los océanos Atlántico y Pacífico con una extensión marítima de aproximadamente $988000 \mathrm{~km}^{2}$. 
Colombia es un país autosuficiente en su aspecto energético. De acuerdo a la información publicada en el la Agencia internacional de energía para el año 2014 la Oferta Primaria Total De Energía (TPES por sus siglas en inglés) ${ }^{1}$ alcanzó los 34008 ktoe (miles de toneladas de petróleo equivalente por sus siglas en inglés) o lo que es lo mismo 1.42 EJ (Ver gráfica 1). Este resultado proviene de una tendencia creciente que se ha manifestado desde hace más de 4 décadas (con una pequeña interrupción a finales de los 90's) (IEA, 2016).

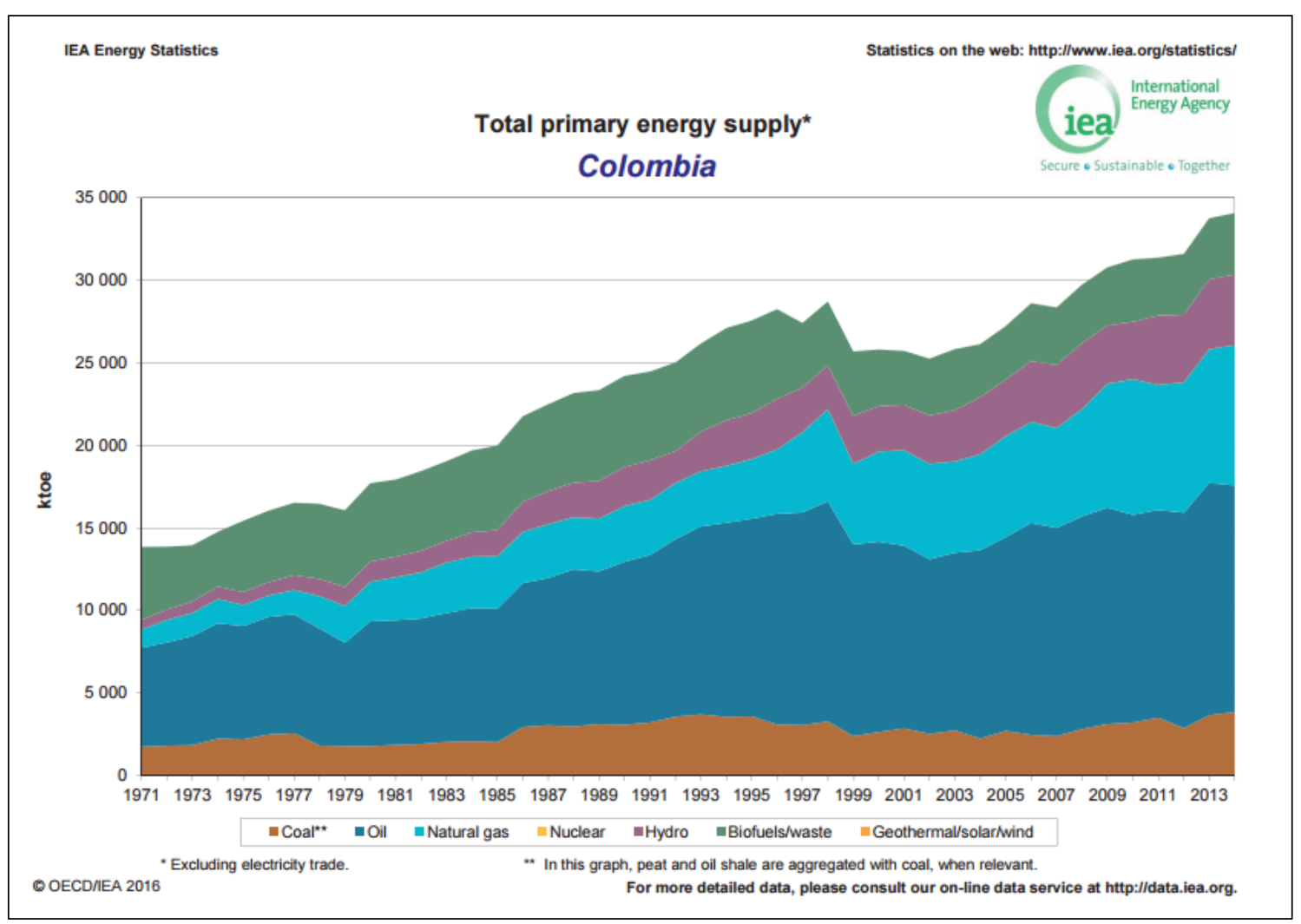

Gráfica 1. Fuente: (IEA, 2016)

En la gráfica 2 se puede ver que la principal fuente energética a lo largo de este período se ha mantenido estable y a constituye la provisión petrolera, que para el año 2014 superó el 40\% del total colombiano. Otras fuentes fósiles, como el gas natural (25\%), y el carbón (11\%) son también importantes para el cubrimiento de la oferta. La oferta energética dada por fuentes renovables (hidroeléctrica, biomasa y desperdicios), cubre un $23 \%$ del total.

\footnotetext{
${ }^{1}$ Nótese que el resultado presentado es neto, ya que las exportaciones son restadas a la producción bruta.
} 


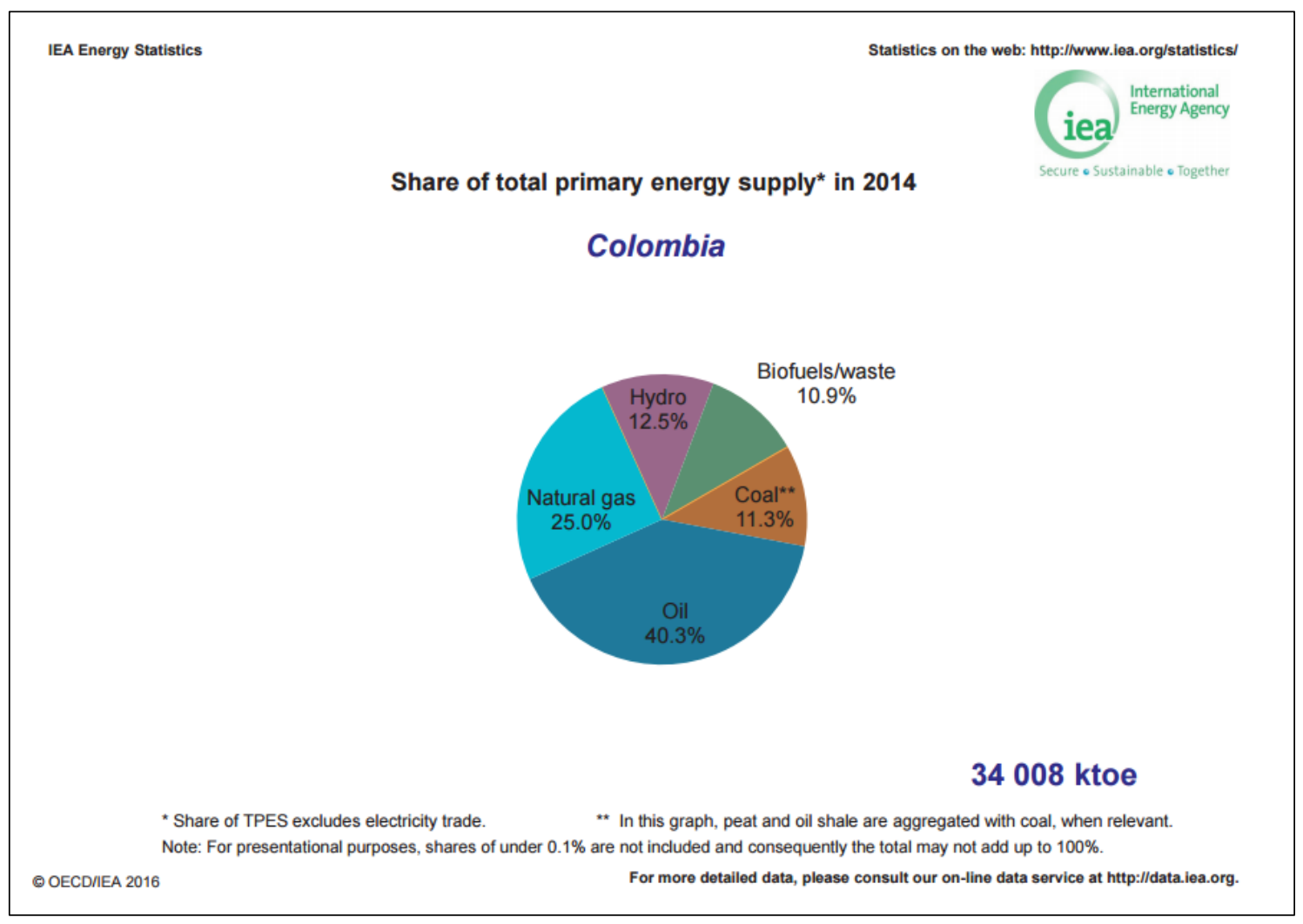

Gráfica 1. Fuente: (IEA, 2016)

Para el mismo año, como se puede apreciar en la gráfica 3, su demanda, dada por el consumo interno de energía, logró los 25635 ktoe (1.07 EJ). El uso más frecuente fue dado por el sector transporte (30\% con más de $10 \mathrm{mil}$ ktoe). El segundo renglón está ocupado por la industria (19\%, 6408 ktoe), seguido por los hogares (14\%, 4951 ktoe). Las aplicaciones energéticas para uso agrícola y de manejo de servicio público ocupan el $6 \%$ y $5 \%$ respectivamente. Otras especificaciones en conjunto dan cuenta de un $26 \%$ aproximadamente. 


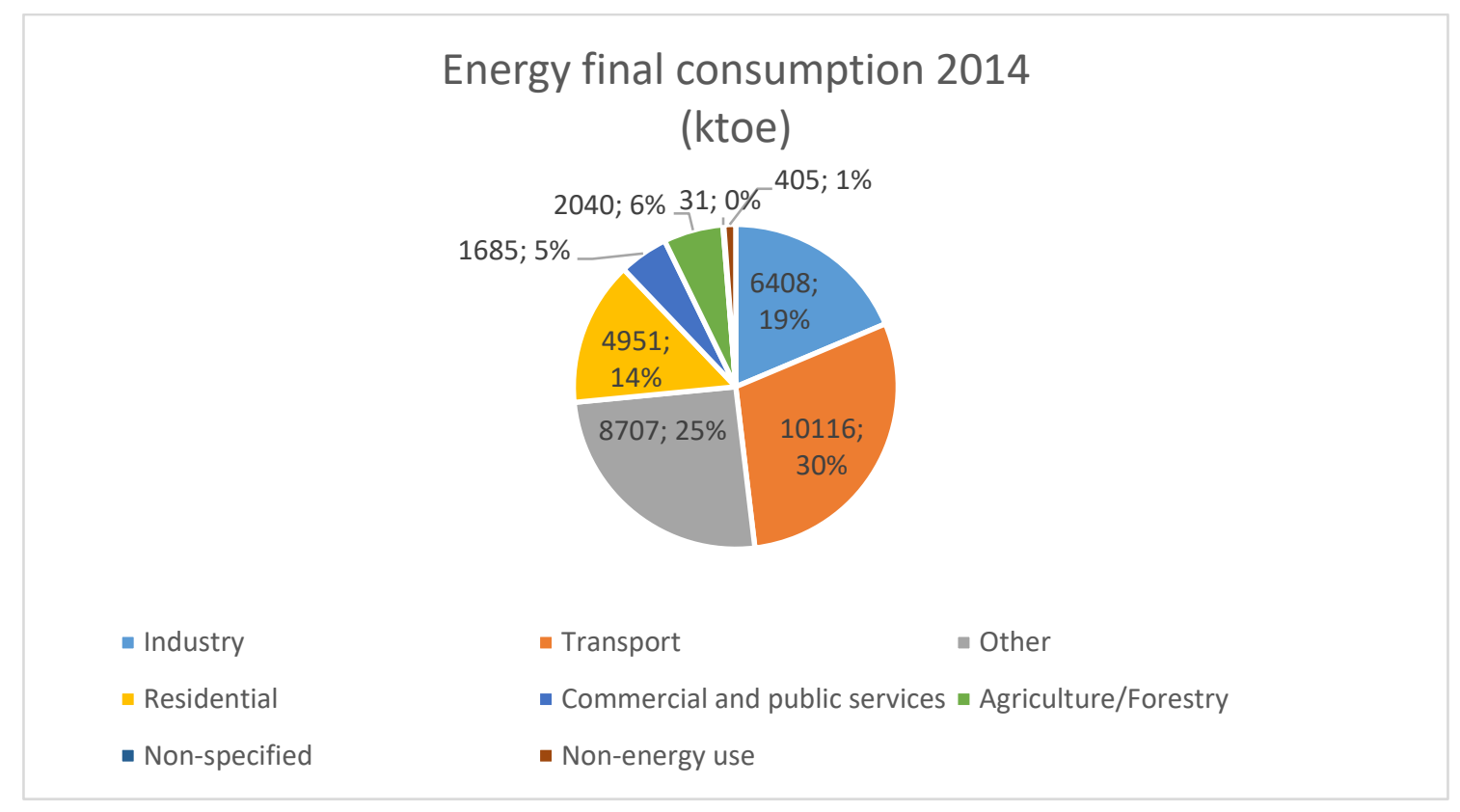

Gráfica 3. Elaboración propia. Fuente de datos: (IEA, 2016)

Sin embargo, el nivel de aprovechamiento energético está circunscrito no solamente a la provisión y sus fuentes, sino que depende en gran medida del uso final que se le quiera dar al recurso como tal. Como se menciona en Ramírez: "por ejemplo, la luz que usada para leer este [texto] es el resultado de la transformación de energía eléctrica en lumínica, que a su vez fue precedida por la transformación de energía potencial -acumulada en un embalse- en energía eléctrica, liberada a través de una turbina hidroeléctrica" (Ramírez Triana, 2010). Algunos expertos en energía como Dale que la energía puede ser aplicada en 3 grandes frentes de acción, a saber: calefacción, movilidad y electricidad (Dale, 2007).

La provisión de calor en los hogares, para fines habitacionales y de comodidad es una necesidad explícita en lugares no-tropicales, sin embargo, para el caso colombiano se aplica el uso de calor para la cocción de alimentos primordialmente. En la industria se usa para fines de generación de potencia y/o electricidad a partir de generadores bajo el principio de Faraday-Lenz. Estos termogeneradores pueden ser alimentados directamente por carbón mineral y/o desperdicios o fuentes de biomasa. Así mismo el gas natural en una opción frecuente para suplir esta necesidad.

Algunas aplicaciones marginales de energía solar térmica se usan para propósitos como calentamiento de agua y bioclimatización a menor escala. Otras iniciativas como la energía solar de concentración empiezan hasta ahora a ser comercializadas en el país. Hay también adelantos recientes en aprovechamiento de biogás, a través de biodigestores en donde se puede 
aprovechar el producto directamente para calefacción o indirectamente para la generación de electricidad.

En el caso de la movilidad, Colombia se soporta fuertemente en gasolina y diésel. Bajo la normatividad actual estos 2 tipos de combustibles se trabajan de forma mezclada con etanol de caña y biodiesel de palma respectivamente, en combinaciones de al menos $5 \%$ de biocombustible y el resto de origen fósil. La flota automotriz que se mueve propulsada por energía eléctrica es aún incipiente como para verse reflejada en los indicadores energéticos nacionales.

Finalmente, la electricidad requiere un análisis aparte. Resulta el tipo de energía más versátil, ya que puede ser aplicada en todos aquellos artefactos (industriales o domiciliarios) que puedan ser conectados a un tomacorriente.

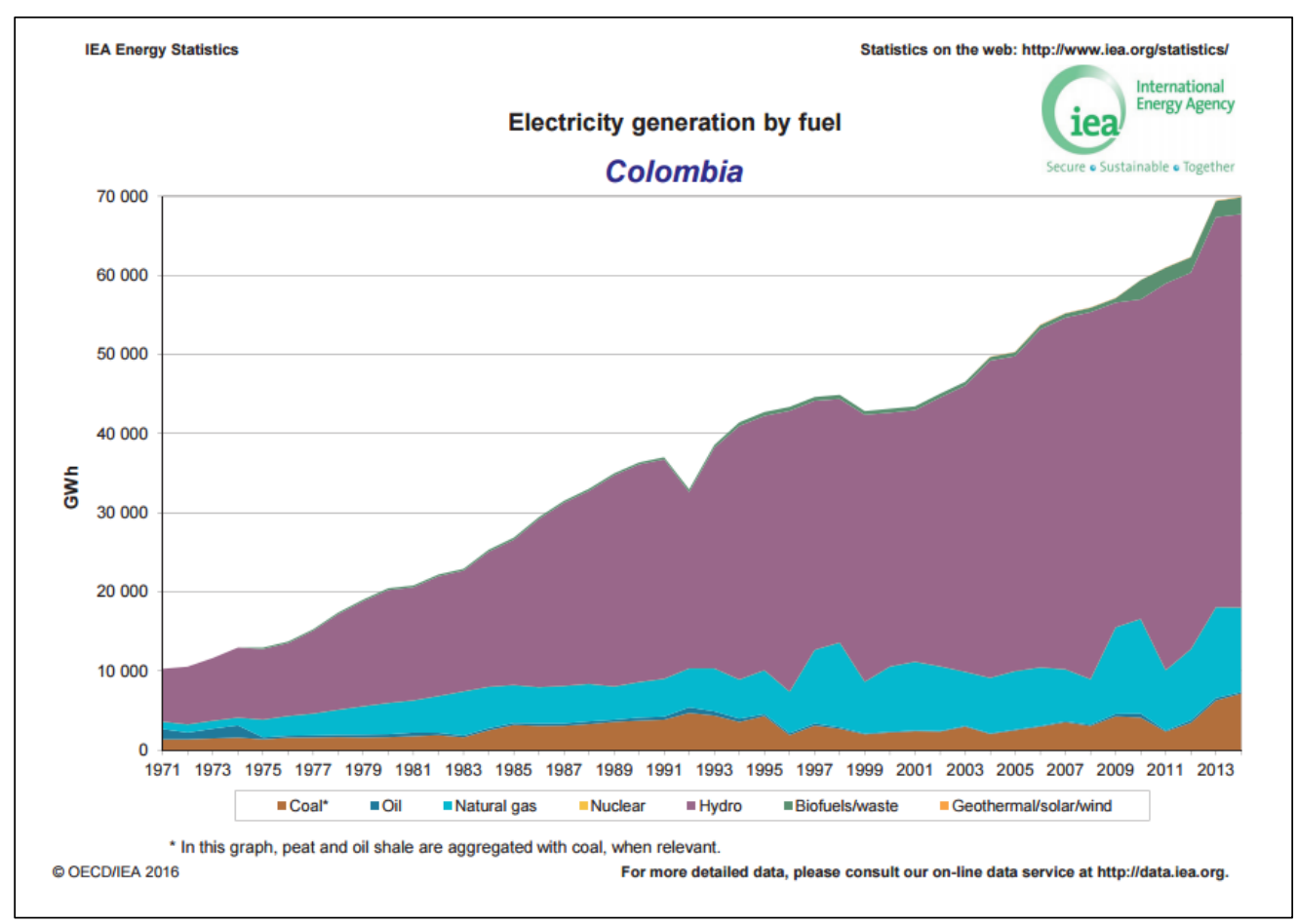

Gráfica 4. Elaboración propia. Fuente de datos: (IEA, 2016)

La fuente principal de energía eléctrica en el territorio nacional, desde hace varias décadas, está dada por proyectos hidroeléctricos. Otras fuentes secundarias de electricidad están respaldadas por recursos fósiles (carbón y gas natural), haciendo uso de termogeneradores, como se indicó anteriormente (Ver gráficas 4 y 5). Es importante resaltar que poco a poco alternativas renovables, soportadas en biomasa van ganando espacio en la provisión de electricidad, a través 
de sistemas de co-generación; mientras que la electricidad basada en petróleo está prácticamente en desuso desde la mitad de los 70's.

\section{Electricity Supply by source 2014}

(GWh;\%)

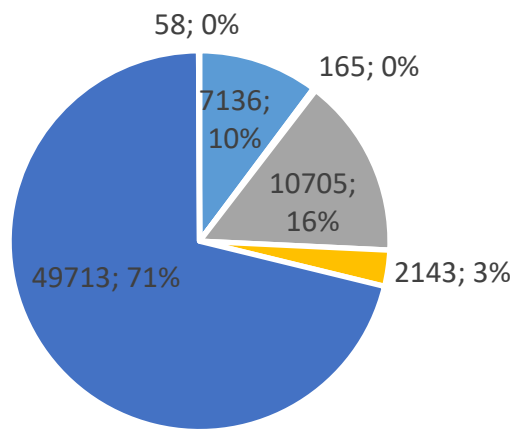

Gráfica 5. Elaboración propia. Fuente de datos: (IEA, 2016)

Ahora, el uso que se le da a esta electricidad está resumido en la gráfica 6, donde se puede observar que principalmente la provisión de este tipo de energía suple mayormente necesidades residenciales, seguidas por el uso industrial y comercial.

\section{Electricity consumption by final use 2014 \\ (GWh)}

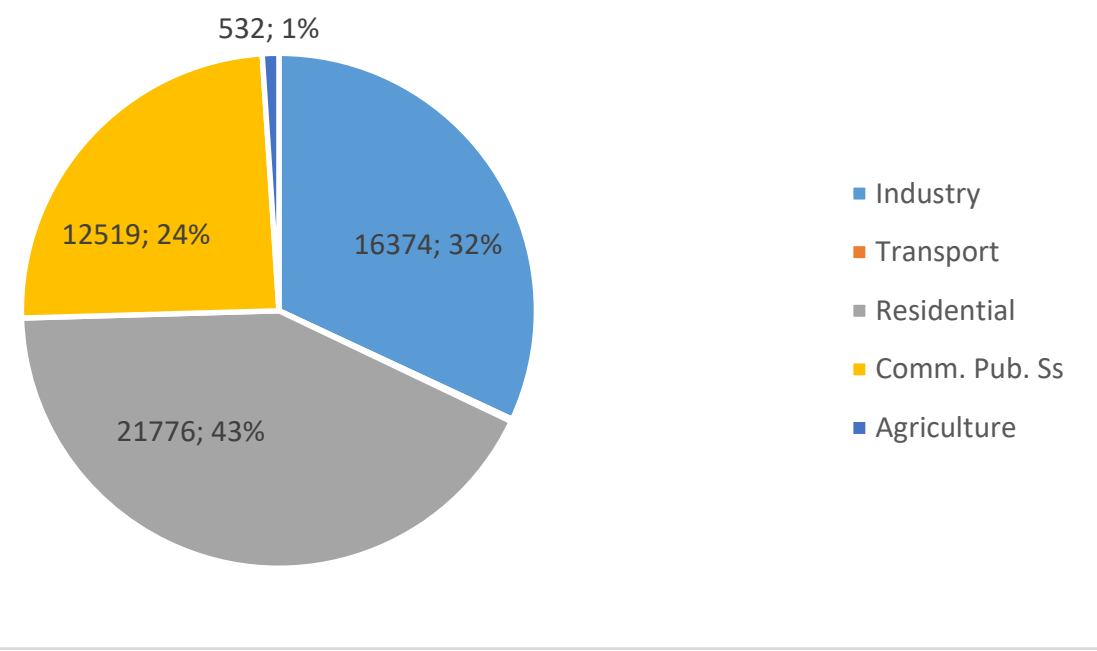

Gráfica 6. Elaboración propia. Fuente de datos: (IEA, 2016)

En menor medida se atienden requerimientos agrícolas. Es precisamente este hecho el que resulta preponderante para lograr entender la importancia de la provisión eléctrica, ya que son los hogares quienes son los principales receptores de este tipo de energía. Dado lo anterior, 
resulta fundamental conocer la cobertura del sistema eléctrico nacional, lo cual se realiza a continuación.

\section{COBERTURA ELÉCTRICA EN COLOMBIA}

En Colombia el Sistema De Información Minero Energético Colombiano (SIMEC), a través del Sistema de información Eléctrico Colombiano (SIEL), se encarga de recopilar la información referente a la provisión de electricidad a lo largo del territorio nacional.

La realidad colombiana en términos de abastecimiento eléctrico es buena, toda vez que se alcanza un índice de cobertura de energía eléctrica (ICEE) cercana a un 97\% de la población. Esto se subdivide en una cobertura del $\mathbf{9 9 . 7 2 \%}$ para los principales asentamientos (conocidos como cabeceras municipales) y de un $87.83 \%$ para el resto del territorio en donde la densidad poblacional es menor (SIEL, 2016).

En un rápido barrido se ve, a partir de las 2 últimas barras de la gráfica 7, que 22 de los 33 departamentos con que cuenta el país cuentan con una cobertura de al menos el $90 \%$ para sus residentes, y en otros 6 la cobertura es de al menos del $80 \%$. Lo cual da un balance positivo de la situación nacional.

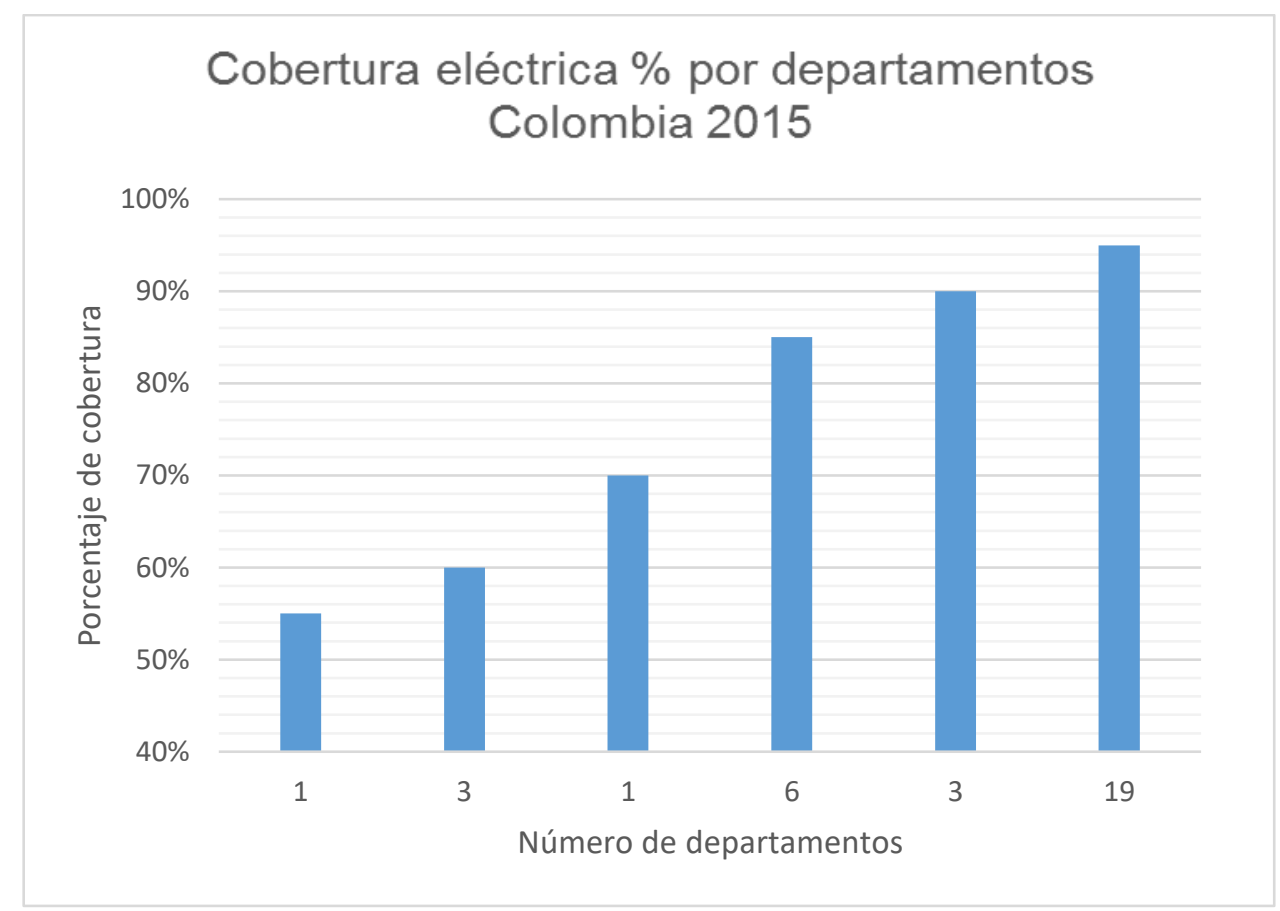

Gráfica 7. Elaboración propia. Fuente de datos: (SIEL, 2016) 
Sin embargo, hay algunas brechas que deben ser reducidas y que enfrentan dificultades importantes en términos de infraestructura y acceso a la malla nacional. Los departamentos más desatendidos (por debajo del 75\% de cobertura), para el año 2015 son La Guajira, Vaupés, Amazonas, Putumayo, Vichada.

A pesar de ser de un momento ligeramente anterior a la presentación de este informe, el siguiente diagrama (gráfica 8) nos permite plantear un panorama de la problemática que se intenta abordar:

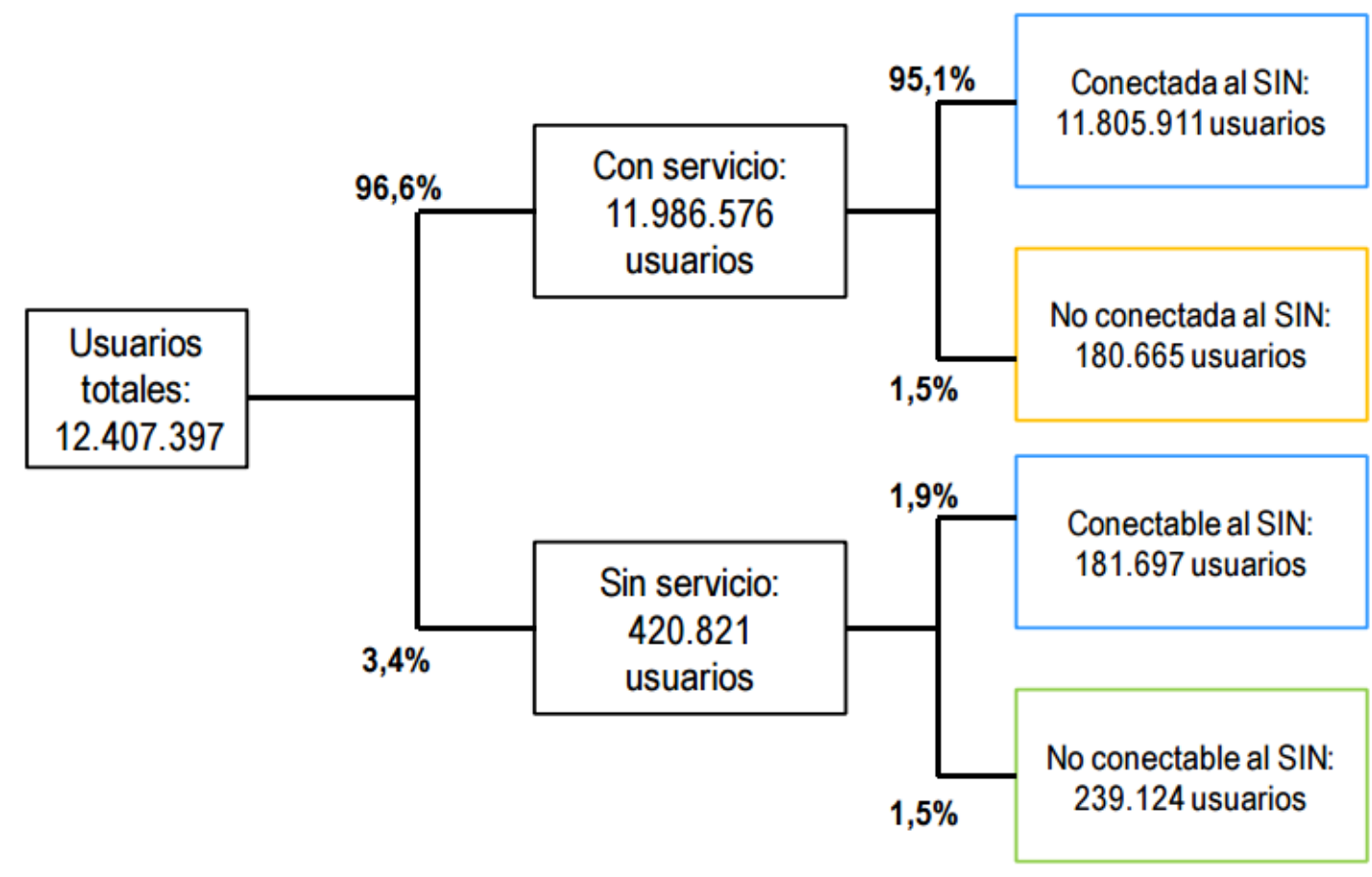

Fuente: UPME PIEC 2010 - 2014

Generación H-T

Generación diesel

Solución aislada

Gráfica 8. Fuente (UPME, 2012)

Los usuarios planteados están dados por el número de hogares registrados en el informe. El pilar fundamental del informe se da alrededor de la sigla ZNI: Zonas No interconectadas. Para todos los efectos relacionados con la prestación del servicio público de energía eléctrica se entiende por Zonas No Interconectadas a los municipios, corregimientos, localidades y caseríos no conectadas al Sistema Interconectado Nacional, SIN (UPME, 2012).

Como se ve en la gráfica 8, se describe la distribución de la población, pero no se limita a registrar a aquellos que cuentan con el servicio y aquellos que no, sino que el primer grupo se subdivide en aquellos que son cubiertos bajo la malla nacional y aquellos que solucionan por sus propios 
medios (no conectados el SIN), básicamente usando plantas generadoras alimentadas con diésel. El segundo grupo, es decir aquellos sin servicio, son clasificados como usuarios potenciales (es decir que sean conectables al SIN) y aquellos que no pueden ser cubiertos por el sistema y que deben buscar otro tipo de solución.

\section{COLOFÓN}

Para dar cierre a este informe preliminar, lo que se pretende es identificar los proyectos que se han implementado para atender las necesidades energéticas de las ZNI. Como en este caso se atienden comunidades aisladas se entiende que la sofisticación y nivel tecnológico de estas soluciones no es muy amplio y puede ser un espacio adecuado para implementar el estudio de la innovación ligera a través de la metodología de los efectos olvidados.

Para lo anterior es importante realizar una descripción completa del aparato institucional encargado de atender este frente, revisar los proyectos implementados hasta la fecha, e identificar causas y consecuencias de la problemática planteada para proceder al planteamiento del cuestionario que se le presentará a los expertos en el tema para poder realizar la cuantificación de los efectos olvidados.

\section{Referencias}

Dale, B. (2007). Thinking clearly about biofuels: ending the irrelevant 'net energy'debate and developing better performance metrics for alternative fuels. . Biofuels, Bioproducts and Biorefining, 14-17.

IEA. (2016, 10 19). Statistics on Colombia: Indicators for 2014. Retrieved from https://www.iea.org/statistics/statisticssearch/report/?year=2014\&country=Co lombia\&product=Indicators

Ramírez Triana, C. A. (2010). Biocombustibles: seguridad energética y sostenibilidad. Conceptualización académica e implementación en Colombia. Punto de Vista, 45-70.

Ramirez Triana, C. A. (2012). Establecimiento De Una Industria Bioenergética Sostenible En Colombia. Cap\&Cua, 1-15.

SIEL. (2016, 10 15). Cobertura de Energía Eléctrica a 2015. Retrieved from Consulta de estadísticas.:

http://www.siel.gov.co/Inicio/CoberturadelSistemalntercontecadoNacional/Co nsultasEstadisticas/tabid/81/Default.aspx 